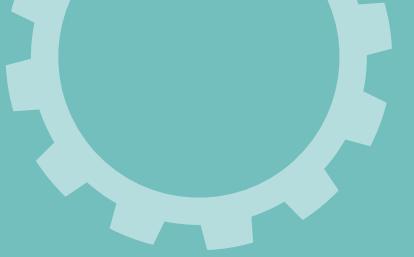

\title{
TURNING BREATH INTO WORDS
}

A new device which transforms paralysis victims' breath into words - believed to be the first invention of its kind - has been developed by academics from

Loughborough University.

Billed as a tool to help bring back the art of conversation for sufferers of severe

paralysis and loss of speech, the prototype analyses changes in breathing patterns

and converts 'breath signals' into words using pattern recognition software and an

analogue-to-digital converter. A speech synthesizer then reads the words aloud.

The Augmentative and Alternate Communication (AAC) device is designed for

patients with complete or partial loss of voluntary muscle control who don't have

the ability to make purposeful movements such as sniffing or blinking - gestures

which previous AAC devices have come to rely upon.

Dr David Kerr, Senior Lecturer in the School of Mechanical and

Manufacturing Engineering, and Dr Kaddour Bouazza-Marouf, Reader in

Mechatronics in Medicine, said the device learns from its user, building up

its knowledge as it goes. It allows the user to control how he or she wishes

to communicate - effectively enabling them to create

their own language by varying the speed of their

breathing. The academics have been joined

in the project by Dr Atul Gaur, Consultant

Anaesthetist at Glenfield Hospital.

'What we are proposing is a system

to form an effective vocabulary that

the machine, said Dr Kerr.
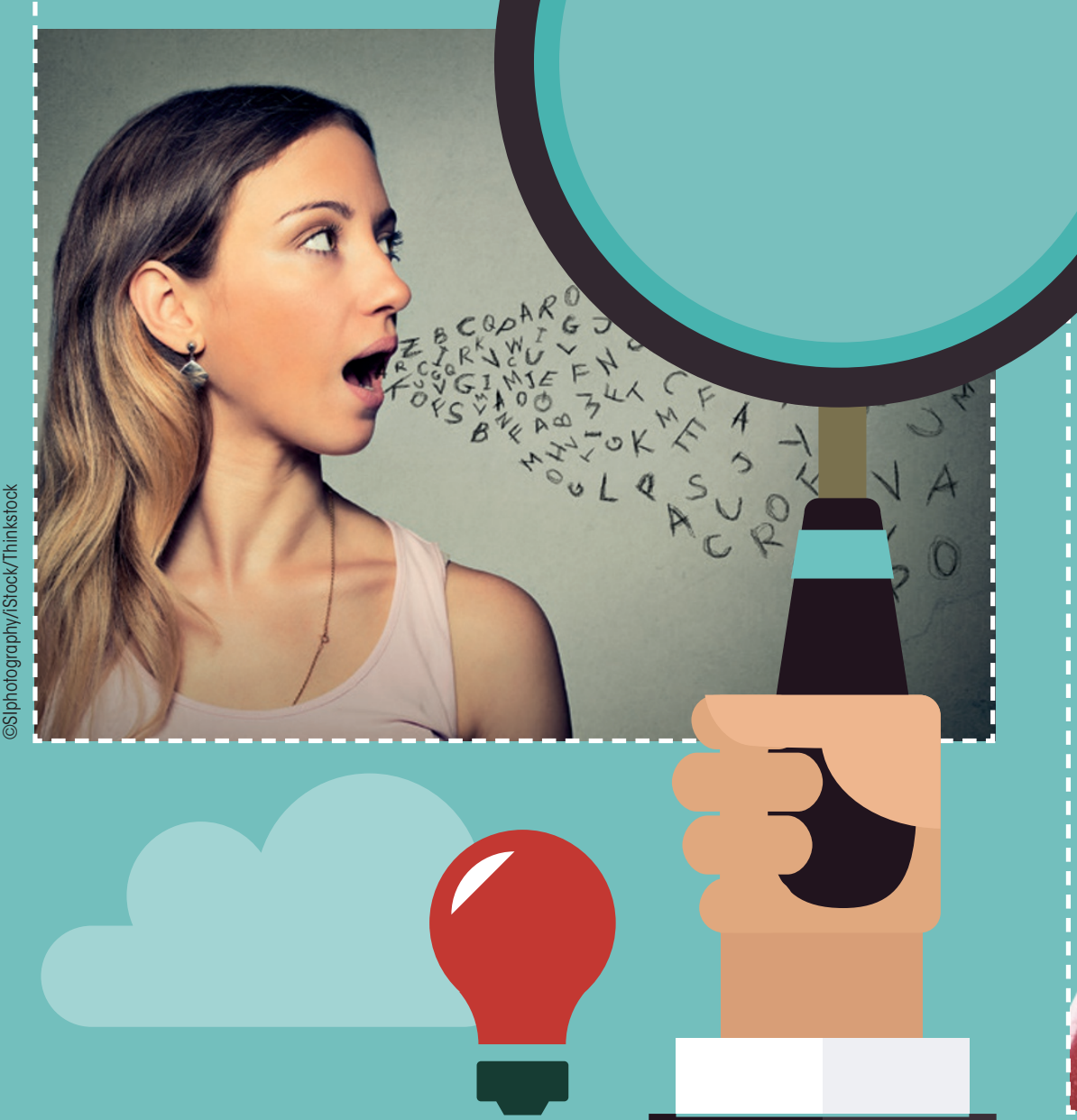

0

\section{FIZZY DRINKS ARE POTENTIAL KILLERS}

Carbonated beverages are associated with out-of-

hospital cardiac arrests of cardiac origin, according

to results from the All-Japan Utstein Registry

presented for the first time today at a congress.

The study in nearly 800000 patients suggests that

limiting consumption of carbonated beverages may be beneficial for health.

'Carbonated beverages, or sodas, have frequently

been demonstrated to increase the risk of

metabolic syndrome and cardiovascular disease

(CVD), such as subclinical cardiac remodeling and stroke. However, until now the association

between drinking large amounts of carbonated

beverages and fatal CVD, or out-of-hospital cardiac arrests (OHCA) of cardiac origin,

was unclear,', said principal investigator

Professor Keijiro Saku, Dean and professor of cardiology at Fukuoka University in Japan.

The analysis focused on the 785,591

OHCA cases that received resuscitation,

of which 435,064 (55.4\%) were of cardiac origin and 350,527 (44.6\%) were of non-

cardiac origin. Those of non-cardiac origin included cerebrovascular disease, respiratory disease, malignant tumour, and exogenous disease $(4.8 \%, 6.1 \%, 3.5 \%$, and $18.9 \%$, respectively).

The researchers found that expenditures on carbonated beverages were significantly associated with OHCAs of cardiac $(\mathrm{r}=0.30, \mathrm{p}=0.04)$, but not

non-cardiac origin $(\mathrm{r}=-0.03, \mathrm{p}=0.8)$.

Expenditures on other beverages, including green tea, black tea, coffee, cocoa, fruit or vegetable juice, fermented milk beverage, milk and mineral water were not significantly associated with OHCAs of cardiac origin.

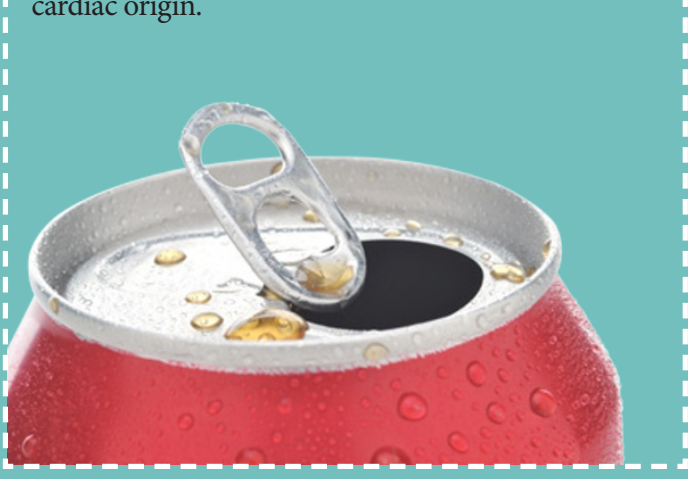

OScukrov/iStock/Thinkstock 